Memorias del VII Encuentro Nacional de Experiencias en la Enseñanza de la Biología y la Educación Ambiental y II Congreso Nacional de Investigación en la Enseñanza de la Biología

\title{
MICROINVERNADEROS COMO ESTRATEGIA DE APROPIACIÓN Y CONSERVACIÓN DE PLANTAS NATIVAS Y ESPACIOS VERDES EN LOS ESTUDIANTES DE GRADO OCTAVO DEL GIMNASIO EL PORTILLO
}

\section{MICROINVERNADEROS AS A STRATEGY OF APPROPRIATION AND CONSERVATION OF NATIVE PLANTS AND GREEN SPACES IN EIGHTH GRADE STUDENTS THE GIMNASIO EL PORTILLO}

\author{
Gineth Adriana Calderón Padilla ${ }^{1}$ \\ Mayra Alejandra Cañizares Martínez ${ }^{2}$ \\ JinethKatherin Castellanos Garzón ${ }^{3}$
}

\section{Resumen}

La vegetación nativa ha cumplido un papel importante a través del tiempo en el desarrollo e identidad de las comunidades. Esta no se limita estrictamente a una función biológica o ecológica en un ecosistema; sino que también interviene en aspectos culturales y sociales de la población humana. A pesar de ello, en la actualidad se ha visto que existe una falta de apropiación por los espacios verdes y un desconocimiento de la función e importancia de las plantas de origen nativo en nuestro entorno. El objeto del presente artículo fue propiciar una conciencia ecológica en los estudiantes de grado octavo del colegio Gimnasio El Portillo del municipio de Cota-Cundinamarca, a partir del reconocimiento de la flora del lugar y la construcción de micro-invernaderos de plantas nativas, lo cual se logró mediante la implementación de seis actividades guiadas, que permitieron dar cuenta de las concepciones de los estudiantes y construir nuevo conocimiento escolar, al complejizar el concepto que tenían hacia los espacios verdes y la flora nativa y exótica desde su propio contexto; y generar sentido de apropiación y conservación de los mismos. Concluyéndose que la siembra de plantas nativas en las zonas verdes de su entorno hace que los estudiantes tengan un contacto más cercano con la flora de su país y puedan apropiarse de sus espacios y tomar conciencia de la importancia de sus usos, así como la implicación que tiene su permanencia o desaparición para un ecosistema.

Palabras claves: Vegetación exótica y nativa, Espacios verdes, Función ecológica, Propagación, Apropiación y Conservación.

\footnotetext{
${ }^{1}$ Universidad Distrital Francisco José de Caldas, Facultad de Ciencias y Educación, Proyecto Curricular de Licenciatura en Biología.

${ }^{2}$ Universidad Distrital Francisco José de Caldas, Facultad de Ciencias y Educación, Proyecto Curricular de Licenciatura en Biología.

${ }^{3}$ Universidad Distrital Francisco José de Caldas, Facultad de Ciencias y Educación, Proyecto Curricular de Licenciatura en Biología.
} 
Memorias del VII Encuentro Nacional de Experiencias en la Enseñanza de la Biología y la Educación Ambiental y II Congreso Nacional de Investigación en la Enseñanza de la Biología

\section{Abstract}

Native vegetation has played an important role over time in the development and identity of communities. This is not strictly limited to a biological or ecological role in an ecosystem, if not also involved in cultural and social aspects of the human population. However, today it is seen that there is a lack of ownership by green spaces and an ignorance of the role and importance of native plants in our surroundings origin. The purpose of this article was foster environmental awareness in the eighth grade students of the school Gym Township Portillo Cota, Cundinamarca, from the recognition of the local flora and the construction of microgreenhouses plant nurseries. This was achieved by implementing guided six activities that allowed accounting for the views of students and build new school knowledge, to complicate their concept of the greenery and native and exotic flora from their own context, and generate ownership and conservation thereof. Conclusion is that the planting of native plants in the green areas of the environment makes students have closer contact with the flora of their country and to seize their spaces and become aware of the importance of its applications and the implication that has its permanence or disappearance to an ecosystem.

Keywords: Exotic and native vegetation, Green Spaces, Ecological function, Spread, Ownership and Conservation.

\section{Introducción}

Las actividades diarias que muchas veces son realizadas de modo inconsciente hacen que se desprecie lo que se tiene alrededor y se ignore la importancia y relación que esto puede tener para la comunidad, como por ejemplo la vegetación que puede proporcionar beneficios tanto en el ecosistema, como en la nutrición y salud humana.

La mayoría de las plantas que se ignoran en un lugar son nativas, las cuales se desarrollan en las áreas biogeográficas de donde son originarias (Tomado de http://www.ecoindustria.org.ar/pdf/Plantas\%20Nativas.pdf el 5 de junio de 2013) y que además aportan gran cantidad de usos que son desconocidos por las personas que frecuentan las zonas en donde se ubican, pero también se pueden observar de tipo exótico que se refiere a las que han sido introducidas en otras partes del mundo diferente a las de su lugar de origen, principalmente son plantas ornamentales y algunas de ellas se han vuelto invasoras, afectando otras especies, ya que no permiten su crecimiento o acidifican los suelos. (Tomado de http://www.ecured.cu/index.php/Planta_ex\%C3\%B3tica el 5 de junio de 2013) 
Memorias del VII Encuentro Nacional de Experiencias en la Enseñanza de la Biología y la Educación Ambiental y II Congreso Nacional de Investigación en la Enseñanza de la Biología

Una manera para propiciar el interés en las personas sobre las plantas nativas y darlas a conocer es mediante el acercamiento y propagación, que a su vez hace que se dé una apropiación de la flora nativa y un buen uso de esta. Una forma para propagar fácilmente es el micro-invernadero que es una técnica que puede realizarse en casa o en colegios en el que se pueden plantar todo tipo de semillas, es cómodo para transportar, no ocupa mucho espacio y puede tener variedad de formas, al poderse realizar en distintos materiales que son considerados como basuras.(Tomado de http://emooziiiioo.blogspot.com/ el 5 de junio de 2013)

Dentro de las plantas nativas que son de gran importancia para recuperación del suelo, evitar la erosión de este o para alimentación, tenemos el chocho de árbol, el lupino, el arrayán, el ají, el moquillo, entre otras.Debido a lo anterior se decidió llevar a cabo en la institución Gimnasio El Portillo un proyecto sobre la propagación de plantas nativas por medio de micro-invernaderos realizados en cubetas de huevos. En este centro educativo ubicado en Cota, Cundinamarca se observó que los estudiantes desconocían las zonas verdes de su colegio, además no participaban de la huerta escolar, ya que no les era permitido, siendo la persona encargada del mantenimiento de la institución quien administraba los cultivos de papa y maíz que habían en esta; además no tenían clara la diferencia entre especies nativas y exóticas y el concepto que poseían estaba ligado a lo que escuchaban y veían en los medios de comunicación; también se hizo evidente que no existía apropiación de la vegetación que les rodeaba, ya que no tenían conocimiento acerca de lo que está plantado en el Gimnasio.

A partir de estas problemáticas y situados dentro del paradigma interpretativo o hermenéutico, se planteó propiciar en los estudiantes una conciencia ecológica, a través del reconocimiento de la flora encontrada en el colegio y la elaboración de micro-invernaderos en donde se sembraron especies nativas, para lograr esto en los jóvenes de octavo grado, se hizo una observación de la vegetación que se estaba en el Gimnasio el Portillo, mediante un recorrido dirigido, con el fin de generar una apropiación de la flora nativa y diferenciarla de la exótica, así como el entendimiento de la implicación que tienen en el ecosistema, lo que dio lugar a que los estudiantes propagaran algunas especies nativas, entendieran la importancia de conservarlas y de hacer buen uso de estas.

Este proyecto fue de gran importancia, puesto que hizo que los estudiantes reconocieran y se apropiaran de los espacios verdes de su colegio, además generó una conciencia sobre la necesidad de conservar las plantas nativas y la 
Memorias del VII Encuentro Nacional de Experiencias en la Enseñanza de la Biología y la Educación Ambiental y II Congreso Nacional de Investigación en la Enseñanza de la Biología

importancia de propagarlas para mantener la especie; estas actividades hicieron que los estudiantesentendieran la diferencia entre una especie nativa y una exótica y cambiaran el imaginario acerca de estas.

\section{Metodología}

La experiencia pedagógica es un producto derivado de un proceso investigativo en el cual, teniendo como referente el problema planteado, los instrumentos diseñados para el mismo y el marco conceptual de apoyo, se adopta una posición desde el paradigma interpretativo o hermenéutico, que según Sánchez (2011) señala que este 'sucede como una interpretación minuciosa, detallada y crítica sobre los aspectos intrínsecos de cada estudiante, el cual se basa en la percepción y las representaciones de cada actor en el proceso de la educación' es por esto que sus datos son bastante específicos y obedecen a la relaciones que pueden transformar a los actores estudiados, es decir no es generalizable en otros contextos. Los cambios actitudinales y las acciones de los sujetos son el eje focal de este paradigma, pues es de ahí que toma los elementos para desarrollar su investigación.

Por ende el contexto particular trabajado bajo este paradigma, se desarrolló en el marco geográfico que se encuentra delimitado dentro del Departamento de Cundinamarca, Municipio de Cota; en el Colegio Gimnasio El Portillo, el cual queda ubicado en el Kilometro 6 Vía Suba-Cota en la vereda El Chorrillo. El Colegio cuenta con grandes extensiones rurales, lo cual le permite considerarse de tipo campestre, por sus características y el aislamiento urbano, es por ello que los espacios que brinda esta institución permitieron el desarrollo de las siguientes actividades realizadas con 22 estudiantes de grado octavo destacadas a continuación en la Tabla 1.

\begin{tabular}{|c|l|l|}
\hline $\begin{array}{c}\text { Cla } \\
\text { se }\end{array}$ & \multicolumn{1}{|c|}{ Actividad } & \multicolumn{1}{c|}{ Apropiación investigativa } \\
\hline 1 & $\begin{array}{l}\text { Cartografía social. } \\
\text { (hagamos memoria) }\end{array}$ & $\begin{array}{l}\text { Por medio de la organización de siete grupos de trabajo con los } \\
\text { estudiantes de grado octavo, se realizaron mapas } \\
\text { representativos de los espacios de la institución académica } \\
\text { según las percepciones y los consensos de los estudiantes. }\end{array}$ \\
\hline 2 & Clase introductoria. & $\begin{array}{l}\text { Para el correcto desarrollo de las actividades planteadas para } \\
\text { este proyecto, fue de vital importancia enmarcar y contextualizar } \\
\text { a los estudiantes sobre la temática a trabajar. Para ello se } \\
\text { identificaron las ideas previas de los estudiantes acerca del }\end{array}$ \\
\hline
\end{tabular}


Memorias del VII Encuentro Nacional de Experiencias en la Enseñanza de la Biología y la Educación Ambiental y II Congreso Nacional de Investigación en la Enseñanza de la Biología

\begin{tabular}{|c|c|c|}
\hline & & $\begin{array}{l}\text { concepto de plantas nativas, exóticas y su importancia ecológica } \\
\text { y económica. }\end{array}$ \\
\hline 3 & $\begin{array}{l}\text { Recorrido guiado por el } \\
\text { colegio.(Reconozcamos } \\
\text { lo que tenemos) }\end{array}$ & $\begin{array}{l}\text { Con la finalidad de que los estudiantes identificaran y } \\
\text { reconocieran la flora nativa y exótica presente en el colegio, se } \\
\text { realizó un recorrido por las zonas verdes presentes allí. Durante } \\
\text { el transcurso del trayecto los siete grupos previamente } \\
\text { organizados, eligieron dos plantas de su interés para su } \\
\text { posterior consulta acerca de su importancia ecológica y } \\
\text { económica. }\end{array}$ \\
\hline 4 & $\begin{array}{l}\text { Creación de micro } \\
\text { invernaderos.(Generan } \\
\text { do vida) }\end{array}$ & $\begin{array}{l}\text { Para generar un sentido de apropiación con la flora nativa de } \\
\text { Colombia, se elaboraron por grupos de trabajo micro- } \\
\text { invernaderos en cubetas recicladas de huevo. Estas cubetas } \\
\text { fueron pintadas en la parte inferior de color negro y en la parte } \\
\text { superior se colocó tierra abonada con las siguientes semillas } \\
\text { distribuidas entre ellos. Dentro de las especies sembradas se } \\
\text { encuentran:Lupino (Lupinusbogotensis), Chocho de monte o } \\
\text { Chochito (Erythrinarubrinervia), Ají (Capsicumannuum), Arrayán } \\
\text { (Myrcianthesleucoxyla), Mano de oso (Oreopanaxfloribundum) y } \\
\text { Moquillo (Saurauiascabra). } \\
\text { Para la siembra de las semillas, por cada especie se tuvieron en } \\
\text { cuenta las consideraciones y preparaciones previas de } \\
\text { propagación específicas para cada una. Estas semillas se } \\
\text { obtuvieron del Jardín Botánico de Bogotá y del Sendero } \\
\text { Ambiental de Mogambo. }\end{array}$ \\
\hline 5 & $\begin{array}{l}\text { Creación de fichas } \\
\text { didácticas. (Plasmando } \\
\text { lo aprendido) }\end{array}$ & $\begin{array}{l}\text { Como material de apoyo para los estudiantes, se estableció un } \\
\text { espacio para la construcción de fichas didácticas, con la } \\
\text { información traída de consultas previas, relacionada con las } \\
\text { plantas nativas cultivadas y observadas dentro de las } \\
\text { instalaciones del colegio, al igual que las plantas exóticas de } \\
\text { este lugar. Los parámetros a considerar dentro de las fichas } \\
\text { didácticas fueron: los nombres comunes y científicos de las } \\
\text { plantas, descripción botánica, distribución, importancia y usos. }\end{array}$ \\
\hline 6 & $\begin{array}{l}\text { Socialización.(Hablemo } \\
\text { s de lo nuestro) }\end{array}$ & $\begin{array}{l}\text { Para la retroalimentación de las actividades realizadas en el } \\
\text { transcurso del proyecto, se procedió a una socialización entre } \\
\text { todos los estudiantes de grado octavo, resolviendo dudas e } \\
\text { inquietudes. }\end{array}$ \\
\hline
\end{tabular}

Tabla1. Actividades divididas en seis sesiones, en las que se observa la apropiación investigativa.

\section{Resultados y discusión}

Se presentaron los siguientes resultados a partir de la metodología planteada en el desarrollo de las actividades; inicialmente a través de la cartografía social se evidenció que los estudiantes manifestaron un interés exclusivo hacia las zonas recreativas de las instalaciones de la institución, destacando principalmente en sus representaciones gráficas las canchas deportivas, seguido de los salones y la cafetería. Lo cual evidencia que los estudiantes solo conciben los espacios deportivos como zonas verdes, mostrando que no tienen un sentido de 
Memorias del VII Encuentro Nacional de Experiencias en la Enseñanza de la Biología y la Educación Ambiental y II Congreso Nacional de Investigación en la Enseñanza de la Biología

pertenencia hacia las demás áreas, debido a que son los únicos espacios de los que hacen uso y ven las demás zonas como lugares o espacios decorativos proporcionándole un único valor estético; pero a través de la ejecución de las actividades los jóvenes lograron reconocer otros sectores de su colegio que hacen parte de las zonas verdes y concientizarse de que estos han sido ignorados por ellos y que es necesario recuperarlos como en el caso específico de la huerta.

Posterior a esto en la actividad introductoria, al realizar el rastreo de las ideas previas de los estudiantes, se logró identificar que estos percibían las plantas exóticas como elementos vistosos, decorativos y llamativos; al mencionar por ejemplo frases como "las plantas exóticas son cosas de color muy bonito y llamativo" y "las plantas exóticas son las que son diferentes a las demás y son más bonitas y raras". Lo que indico una percepción distinta a lo que en realidad se cita en la literatura, quizá por la influencia que tienen las actividades que realizan o lo que les comentan sus padres, ya que "hoy en día, los niños están sumergidos en la cultura de los medios de comunicación a través de la televisión, los videos, los juegos por computadoras y la Internet" (Tomado de http://www.educando.edu.do/articulos/familia/influencia-de-los-medios-los-de comunicacin-en-niosas/ el 06 de junio de 2013), lo que tal vez hizo que se creara esta imagen en el pensamiento de los muchachos; sin embargo en la última actividad que trataba sobre la socialización de lo aprendido, se pudo observar que los estudiantes pudieron entender el concepto de una especie exótica, es decir, pasaron de una idea generada por las dinámicas culturales a una de concientización como se ve en esta frase "profe entonces todas plantas que tenemos son exóticas y estamos dañando nuestro colegio" lo que muestra que las actividades anteriores, sobre todo la del recorrido por el colegio fueron importantes para este cambio de percepción.

Con relación a las plantas nativas no se poseía ninguna concepción, ya que no se obtuvo respuesta alguna al momento de indagar sobre ellas.Cabe señalar que se hizo una aclaración acerca de lo que se aprecia como una planta exótica invasora como el ojo de poeta (Thunbergiaalata) y una que no, respecto a su implicación ecológica, lo que generó que los jóvenes tuvieran una visión más clara sobre esta temática y pudieran lograr una apropiación más fuerte por las especies nativas.

Con el desarrollo de la actividad de recorrido guiado los estudiantes al seleccionar las plantas para su respectiva identificación y posterior consulta, tendieron a elegir aquellas más vistosas y coloridas, las cuales en su mayoría 
Memorias del VII Encuentro Nacional de Experiencias en la Enseñanza de la Biología y la Educación Ambiental y II Congreso Nacional de Investigación en la Enseñanza de la Biología

fueron exóticas. Sin embargo, a partir de su consulta estos se dieron cuenta, que en su entorno se encuentran en mayor proporción plantas de origen exótico que nativo.Al iniciar con la actividad de creación de micro-invernaderos, los estudiantes empezaron a expresar su interés en las plantas nativas mencionando frases como "profe entonces todas plantas que tenemos son exóticas y estamos dañando nuestro colegio"; "¿Son mejores entonces las plantas de nosotros para el ambiente?"; ¿Por qué tenemos entonces plantas exóticas si no son tan buenas?". Lo que generó que trabajaran más activamente al momento de realizar la siembra en los micro-invernaderos con las semillas nativas provenientes del Jardín Botánico de Bogotá y del Sendero Ambiental de Mogambo.

Con lo anterior se lograron evidenciar aspectos como que los estudiantes muestran más interés en la clase si se crean espacios en donde puedan interactuar físicamente con la temática; es decir, mostraron mayor interés cuando se hizo la actividad del recorrido guiado y aún más, cuando se elaboraron los micro-invernaderos, lo que confirma que el despertar la curiosidad en los estudiantes ayuda al proceso de enseñanza-aprendizaje, fortaleciendo las relaciones entre el docente y el estudiante y el desarrollo de un conocimiento escolar, así como lo plantea Vasquez y Manassero (2009) quienes afirman que la ciencia curiosa hace que los estudiantes se relacionen, participen y se motiven más por la clase, ya que permite que ellos mismos puedan elegir qué investigar, plantear sus propósitos y llegar así a resultados que posteriormente contrastan con lo teórico, generándose conclusiones, que contribuyen a su construcción de conocimiento.

Por último, para complementar la actividad de siembra los estudiantes realizaron unas fichas didácticas, previo a la socialización final, en donde plasmaron la importancia de las plantas nativas sembradas y observadas en su entorno con el fin de socializarlo posteriormente, realizando paralelos entre las plantas exóticas y nativas de su colegio, y estableciendo la importancia de mantener un sentido de apropiación hacia la vegetación nativa que cumple una función ecológica en el lugar. De esta manera, durante la actividad, los estudiantes mostraron el producto final de los micro-invernaderos, expresando las razones del crecimiento o no de las plantas. Lo cual les permitió dejar de observar las plantas como un elementomás de su colegio, para apreciarlas por sus usos ecológicos, industriales y/o medicinales, un ejemplo fue la discusión sobre la palma de cera, que aunque no estaba en la institución, despertó preocupación en un grupo de estudiantes, 
Memorias del VII Encuentro Nacional de Experiencias en la Enseñanza de la Biología y la Educación Ambiental y II Congreso Nacional de Investigación en la Enseñanza de La Biología

quienes además de elaborar la ficha, concluyeron que las tradiciones religiosas o culturales hacen que las especies.

\section{Conclusiones}

La siembra de plantas nativas hace que los estudiantes tengan un contacto más cercano con la flora de su país y puedan tomar conciencia de la importancia de sus usos, así como la implicación que tiene su permanencia o desaparición para un ecosistema.

Es importante mostrarles a los estudiantes que existen otros espacios fuera del aula escolar que pueden ser vistos como escenarios de aprendizaje y no de decoración; siendo estos lugares más tangible y propicios para la construcción de conocimiento.

Por medio del desarrollo de este proyecto se pudo concluir que los estudiantes presentan diferentes concepciones que van ligadas a sus contextos, y que por ende no son acordes a nivel biológico a los conceptos de lo nativo y lo exótico.

Es necesario generar en las personas un sentido de pertenencia por lo que les rodea para que puedan ser partícipes de forma positiva en todas las dinámicas que se presenten.

\section{Referencias bibliográficas}

Conocimiento con todos y para todos Ecured (2013). Plantas Exóticas. Recuperado de http://www.ecured.cu/index.php/Planta_ex\%C3\%B3tica.

Educando el Portal sobre la Educación Dominicana. (septiembre 1 de 2005). Influencia de los Medios los de Comunicación en Niños/as. [Web Log Post]. Recuperado de http://www.educando.edu.do/articulos/familia/influencia-de-losmedios-los-de-comunicacin-en-niosas/.

Emooziiiioo. (Diciembre 21 de 2011). Pequeño Microinvernadero. [Web Log Post]. Recuperado de http://emooziiiioo.blogspot.com/.

Plantas Nativas (2013). Ecoindustria.org. Recuperado de http://www.ecoindustria.org.ar/pdf/Plantas\%20Nativas.pdf 
Bio-grafia Escritos sobre la Biología y su Enseñanza.

Edición Extra-Ordinaria. ISSN 2027-1034 P.p 467-475

Memorias del VII Encuentro Nacional de Experiencias en la Enseñanza de la Biología y la Educación Ambiental y II Congreso Nacional de Investigación en la Enseñanza de La Biología

Sánchez, J. (2011). Tradiciones Epistemológicas en Investigación Educativa: Paradigmas Clásicos. De las Leyes Subyacentes a la Modernidad Reflexiva. Revista Digital Sociedad de la información. Madrid.

Vázquez, A. y Manassero, M.A. (2009). La Relevancia de la Educación Científica: Actitudes y Valores de los Estudiantes Relacionados con la Ciencia y la Tecnología. Enseñanza de las Ciencias, 27(1), 33-48. 